\title{
Impact of diastolic dysfunction severity on global left ventricular volumetric filling - assessment by automated segmentation of routine cine cardiovascular magnetic resonance
}

Dorinna D Mendoza', Noel CF Codella², Yi Wang ${ }^{2}$, Martin R Prince ${ }^{2}$, Sonia Sethi', Shant J Manoushagian', Keigo Kawaji ${ }^{2}$, James K Min ${ }^{1,2}$, Troy M LaBounty ${ }^{1}$, Richard B Devereux' ${ }^{1}$, Jonathan W Weinsaft ${ }^{1,2^{*}}$

\begin{abstract}
Objectives: To examine relationships between severity of echocardiography (echo) -evidenced diastolic dysfunction (DD) and volumetric filling by automated processing of routine cine cardiovascular magnetic resonance (CMR).
\end{abstract}

Background: Cine-CMR provides high-resolution assessment of left ventricular (LV) chamber volumes. Automated segmentation (LV-METRIC) yields LV filling curves by segmenting all short-axis images across all temporal phases. This study used cine-CMR to assess filling changes that occur with progressive DD.

Methods: 115 post-MI patients underwent CMR and echo within 1 day. LV-METRIC yielded multiple diastolic indices - E:A ratio, peak filling rate (PFR), time to peak filling rate (TPFR), and diastolic volume recovery (DVR 80 proportion of diastole required to recover $80 \%$ stroke volume). Echo was the reference for DD.

Results: LV-METRIC successfully generated LV filling curves in all patients. CMR indices were reproducible ( $\leq 1 \%$ inter-reader differences) and required minimal processing time (175 \pm 34 images/exam, 2:09 \pm 0:51 minutes). CMR E:A ratio decreased with grade 1 and increased with grades 2-3 DD. Diastolic filling intervals, measured by $D R_{80}$ or TPFR, prolonged with grade 1 and shortened with grade 3 DD, paralleling echo deceleration time $(p<0.001)$. PFR by CMR increased with DD grade, similar to E/e' $(p<0.001)$. Prolonged DVR 80 identified $71 \%$ of patients with echoevidenced grade 1 but no patients with grade 3 DD, and stroke-volume adjusted PFR identified $67 \%$ with grade 3 but none with grade 1 DD (matched specificity $=83 \%$ ). The combination of DVR 80 and PFR identified $53 \%$ of patients with grade $2 \mathrm{DD}$. Prolonged $\mathrm{DVR}_{80}$ was associated with grade $1(\mathrm{OR} 2.79, \mathrm{Cl} 1.65-4.05, \mathrm{p}=0.001)$ with a similar trend for grade 2 (OR 1.35, Cl 0.98-1.74, $\mathrm{p}=0.06$ ), whereas high PFR was associated with grade 3 (OR 1.14, $\mathrm{Cl} 1.02-1.25, \mathrm{p}=0.02) \mathrm{DD}$.

Conclusions: Automated cine-CMR segmentation can discern LV filling changes that occur with increasing severity of echo-evidenced DD. Impaired relaxation is associated with prolonged filling intervals whereas restrictive filling is characterized by increased filling rates.

\footnotetext{
* Correspondence: jww2001@med.cornell.edu

'Division of Cardiology, Department of Medicine, Weill Cornell Medical

College, NY, NY, USA

Full list of author information is available at the end of the article
} 


\section{Background}

Left ventricular (LV) diastolic dysfunction (DD) has important consequences following acute myocardial infarction (MI) as heart failure and mortality risks are linked to severity of diastolic impairment [1-3]. DD alters timing and profiles of LV filling, which initially compensate for impaired LV relaxation but ultimately impede LV performance. Echocardiography (echo) identifies DD based on mitral inflow or myocardial compliance sampled at regional myocardial locations $[4,5]$. This approach is potentially limited by localized changes in LV contractility and myocardial tissue composition, affecting regional compliance but not necessarily impacting global diastolic performance $[4,6]$. Global LV filling curves, which have been previously employed using radionuclide imaging techniques such as RNCA and SPECT, provide an alternative means of assessing diastolic physiology based on timing and pattern of dynamic changes in LV chamber volumes [7-10].

Cardiovascular magnetic resonance (CMR) is a standard for LV chamber volumes and ejection fraction (EF) based on quantification of cine images acquired at enddiastole and end-systole $[11,12]$. Cine-CMR acquires dynamic images throughout the cardiac cycle and thereby contains intrinsic data concerning volumetric changes during diastole. Manual segmentation of cineCMR is an impractical means of assessing LV filling as quantification of all slice positions across all temporal phases typically requires planimetry of over 150 images. We have recently developed an automated algorithm (LV-METRIC) that quantifies chamber volumes using routine cine-CMR. In initial validation studies, LVMETRIC agreed with manual planimetry of phantom and clinical volumes $[13,14]$. In another study, LVMETRIC filling curves by LV-METRIC differentiated between binary presence or absence of echo-evidenced DD [15]. However, all patients had normal systolic function, retrospective data was used for validation, and the relation between cine-CMR filling parameters and graded severity of DD was not evaluated.

The current study employed LV-METRIC among a broad post-MI population with variable systolic function. In all patients, dedicated echo imaging was prospectively done within one day of CMR to provide a uniform reference for diastolic performance. The aim of this study was to test whether automated segmentation of routine cine-CMR can discern LV filling changes that occur with graded severity of diastolic impairment.

\section{Methods}

\section{Population}

The population comprised consecutive patients enrolled in a post-MI imaging registry (clinical trial \#NCT00539045) who underwent CMR and echo within one day. Patients were excluded if echo characterization of diastolic performance was incomplete due to absence of tissue Doppler or pulmonary vein inflow patterns $(n=6)$, arrhythmia prohibiting echo/CMR assessment of LV filling $(n=2)$, or intolerance of CMR $(n=1)$. No patients were excluded based on clinical characteristics or CMR processing results.

To minimize the impact of post-MI stunning and transient volume shifts on LV performance [16], imaging was performed during a pre-specified time frame of $20-$ 40 days post-MI. Imaging was completed between August 2005 and December 2009 at Weill Cornell Medical College (WMC). The study was conducted in accordance with the WMC Institutional Review Board; all patients provided written informed consent.

\section{Imaging Protocol \\ Echocardiography}

Transthoracic echoes were performed using commercial equipment (General Electric Vivid-7 or Siemens Sequoia). Echo measurements, adjudication of diastolic filling patterns, and classifications of diastolic grade were made by an experienced echocardiographer (RBD) blinded to CMR results. Mitral inflow parameters and tissue Doppler profiles were acquired in an apical 4chamber view. Linear measurements of chamber size and systolic function were performed in accordance with consensus guidelines [17]. DD was classified based on both mitral inflow and tissue Doppler parameters [4]. Deceleration time cutoffs were in accordance with previously reported criteria and established standards in the WMC echo laboratory $[1,15,18,19]$. Diastolic performance was graded as follows:

- Normal: E/A $\geq 0.8$, septal e' $\geq 8 \mathrm{~cm} / \mathrm{s}$, lateral e' $\geq 10 \mathrm{~cm} / \mathrm{s}$, deceleration time (DT) $140-240 \mathrm{msec}$

- Grade 1 (mild): E/A < 0.8, septal e' $<8 \mathrm{~cm} / \mathrm{s}$, lateral e' $<10 \mathrm{~cm} / \mathrm{s}$, DT $>240 \mathrm{msec}$

- Grade 2 (moderate): E/A 0.8-1.5, septal e' $<8 \mathrm{~cm} / \mathrm{s}$, lateral e' $<10 \mathrm{~cm} / \mathrm{s}$, DT $140-240 \mathrm{msec}$

- Grade 3 (severe): E/A $\geq 2$, septal e' $<8 \mathrm{~cm} / \mathrm{s}$, lateral e' $<10 \mathrm{~cm} / \mathrm{s}$, DT $<140 \mathrm{msec}$

In patients with equivocal tissue Doppler indices (i.e. abnormal lateral, normal septal e' amplitude), e'/a' reversal $(<1)$ and pulmonary vein profiles were used to establish presence of DD $[4,5]$.

\section{CMR}

CMR was performed using 1.5 Tesla scanners (General Electric). Cine-CMR used a commercially available 2D steady state free precession pulse sequence. Images were acquired in contiguous short-axis slices from the mitral 
annulus through the LV apex. Typical parameters were repetition time $3.5 \mathrm{msec}$, echo time $1.6 \mathrm{msec}$, flip angle $60^{\circ}$, in-plane spatial resolution $1.9 \mathrm{~mm} \times 1.4 \mathrm{~mm}$, slice thickness $6 \mathrm{~mm}$, inter-slice gap $4 \mathrm{~mm}$, reconstructed temporal resolution $36.5 \pm 9.2 \mathrm{msec}$. Delayed enhancement CMR (DE-CMR) was performed in all patients for assessment of LV infarct size.

\section{Automated CMR Segmentation LV Systolic Function and Morphology}

Automated quantification of LV volumes and myocardial mass was performed using LV-METRIC. User input included identification of the slice range to be segmented and definition of the mitral and aortic valve annulus. Optional corrections comprised manual contouring to restrict region-growth and adjusting blood sensitivity. $\mathrm{LV}$ volumes were quantified as the sum of short axis chamber volumes (2D area * slice thickness) measured during end-diastole (EDV) and end-systole (ESV). Ejection fraction (EF) was calculated as EDV-ESV]/ EDV*100. Automated border detection of end-diastolic endocardial and epicardial contours quantified LV mass, calculated as the product of myocardial volume and specific gravity ([EpiEDV-EDV]*1.05) [20].

\section{Adjunctive Analyses}

CMR images were analyzed to quantify other structural indices that can impact LV diastolic performance. Left atrial volume was quantified in accordance with guidelines for biplane chamber planimetry [17]. LV wall motion and infarct size were scored using a 17-segment model. Segmental infarction was based on transmural extent of hyperenhanced (bright) myocardium on DE-CMR and graded using a 5 -point scale $(0=$ no hyperenhancement; $1=1-25 \% ; 2=26-50 \% ; 3=51-75 \%$; $4=76-100 \%)$. Global infarct size as a percentage of LV myocardium was calculated by summing the segmental scores (each weighted by the midpoint of the range of hyperenhancement) and dividing by the total number of regions [21].

\section{Diastolic Function}

LV-METRIC segmentation was performed for all LV short-axis slices across all temporal phases (Figure 1A) to assess the time course of global volumetric filling (Figure 1B). Volumetric data were low pass filtered to remove any non-physiologic high frequency components from the volume-filling curve. The purpose of the filter was to suppress small segmentation errors in the volume curve from being amplified in the derivative curve. The following diastolic parameters were evaluated:

- Peak Filling Rate [PFR] - maximal LV filling rate defined by maximal change in LV volume between sequential temporal phases ( $\Delta$ volume/ $\Delta$ phase). This index was also normalized for LV stroke volume [nPFR]
- Time to Peak Filling Rate [TPFR] - time interval between end-systole and peak filling rate

- Diastolic $80 \%$ Volume Recovery [DVR 80 ] - proportion of diastole required to recover $80 \% \mathrm{LV}$ stroke volume, a cutoff based on prior validation data [15]

Figures $1 \mathrm{~B}$ provides representative illustrations of each parameter. In addition, the first derivative of the volume-time curve was used to generate early (E) and late (A) filling profiles, similar to a typical echo mitral inflow pattern (Figure 2).

\section{Statistical Methods}

Comparisons between groups were made using analysis of variance (for multiple group comparisons) or Student's t-test for continuous variables, with results expressed as mean \pm standard deviation. Categorical variables were compared using the Chi-square test. Bivariate correlation was employed to evaluate associations between continuous echo and CMR parameters. Multinomial logistic regression was used to assess relations of CMR parameters to echo DD grade. Two-sided $\mathrm{p}<0.05$ was considered indicative of statistical significance. Statistical calculations were performed using SPSS 12.0 (SPSS Inc, Chicago, IL).

\section{Results}

\section{Population Characteristics}

The population consisted of 115 post-MI patients who underwent echo and CMR within a one day interval (97\% same day); 65\% had echo-evidenced DD. Diastolic dysfunction was mild (grade 1$)$ in $28 \%(n=21)$, moderate (grade 2$)$ in $60 \%(n=45)$, and severe (grade 3$)$ in $12 \%(\mathrm{n}=9)$ of affected patients.

As shown in Table 1, patients with DD were older and more likely to have hypertension than unaffected patients. Both echo and cine-CMR demonstrated that DD was accompanied by progressive systolic dysfunction as measured by LVEF or wall motion score $(p=0.001)$. Consistent with this, DE-CMR demonstrated a stepwise increase in infarct size in relation to graded severity of DD ( $\mathrm{p}=0.005)$. Whereas LV mass was similar between groups, there was a direct relationship between diastolic grade and left atrial size measured by either echo linear dimensions or cine-CMR volumes ( $\mathrm{p} \leq 0.005$ ).

\section{Automated cine-CMR Segmentation}

LV-METRIC successfully generated filling curves in all cases, yielding multiple diastolic parameters (Table 2). Figure 1 provides a representative example of automated cine-CMR segmentation by LV-METRIC (Figure 1A) as well as a resultant LV filling curve (Figure 1B). E and A waves, obtained by taking the first derivative of the volume-time curve, were discernable in $94 \%(n=108)$ of patients. Figure 2 provides typical E:A filling profiles 


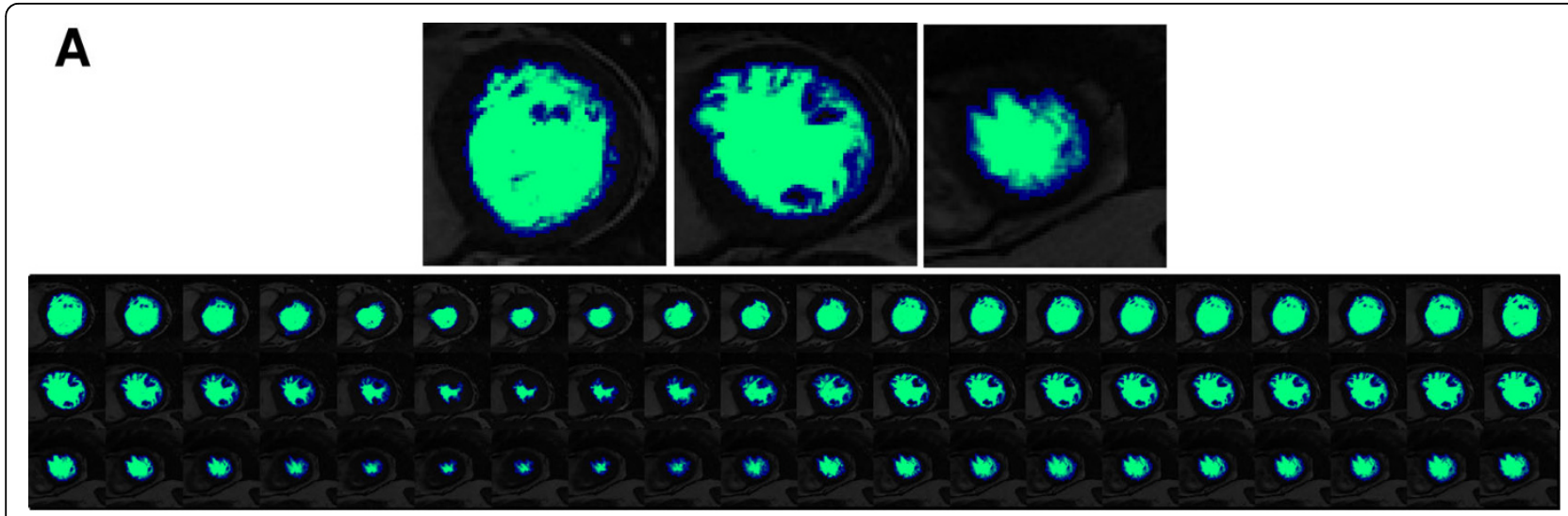

B
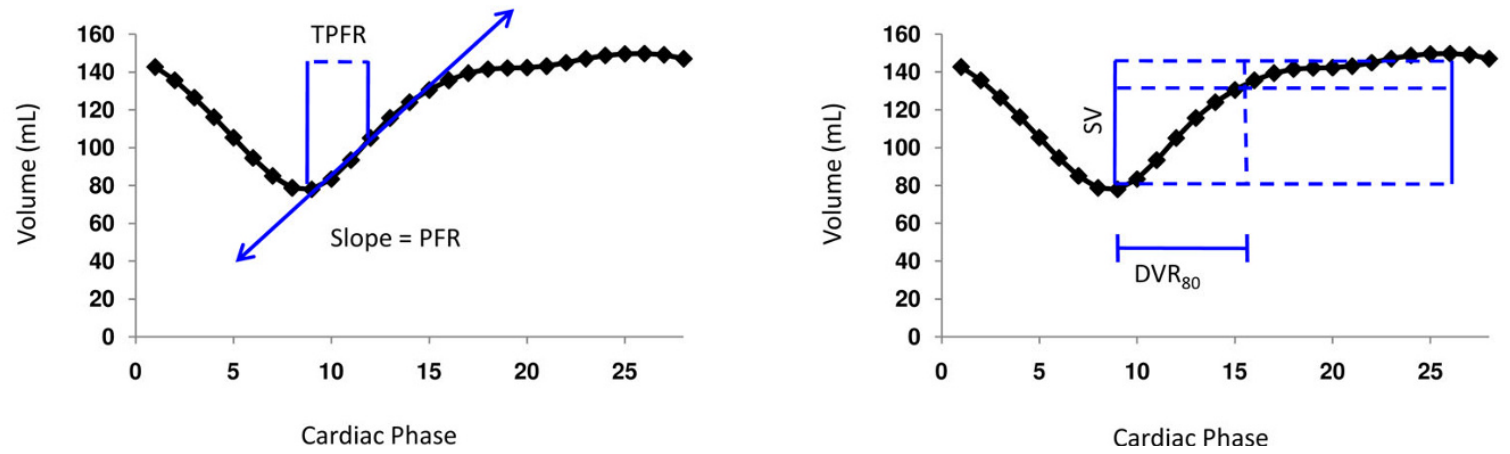

Figure 1 Typical Example of Automated Segmentation. (A) LV-METRIC segmentation of representative images from basal, mid, and apical slice locations (top). Segmentation was performed across spatial (vertical) and temporal (horizontal) domains for volumetric assessment of global LV filling (bottom). (B) Diastolic filling indices generated by LV-METRIC. Peak filling rate (PFR), defined as maximal slope of $\Delta$ volume/ $\Delta$ temporal phase, and time to peak filling rate (TPFR) are shown on the left-sided graph. Diastolic volume recovery (DVR $_{80}$ ), calculated as proportion of diastole necessary to recover a threshold of $80 \%$ LV stroke volume (SV), is shown on the right-sided graph.
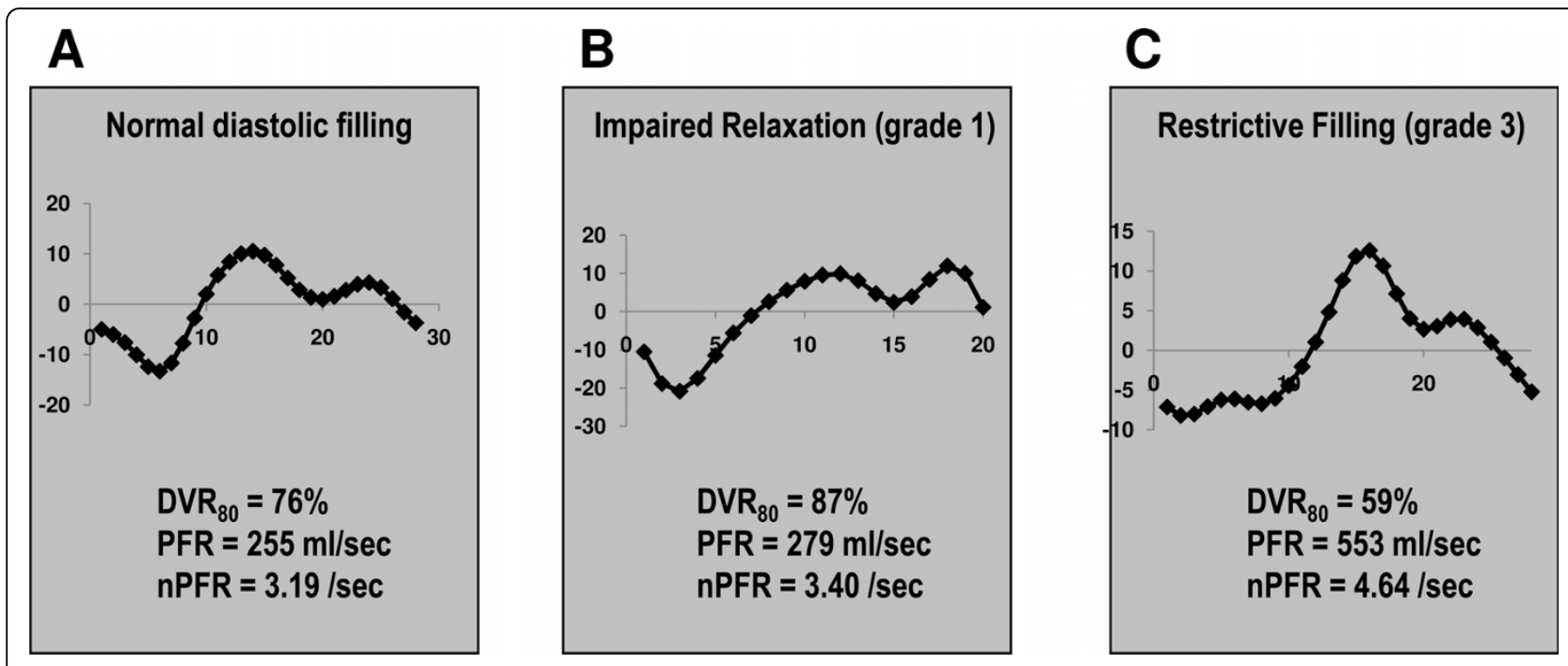

Figure 2 Representative Filling Profiles. Representative E:A profiles generated by cine-CMR for patients with normal echo-evidenced filling (A), grade 1 (B) and grade 3 (C) DD. Note E:A reversal with prolonged DVR 80 for grade 1, and augmented E:A ratio with increased PFR for grade 3 $\mathrm{DD}$. The $\mathrm{x}$-axis represents phase and the $\mathrm{y}$-axis is volume per phase. 
Table 1 Clinical and Conventional Imaging Characteristics

\begin{tabular}{|c|c|c|c|c|c|}
\hline & $\begin{array}{l}\text { Normal } \\
\text { Diastolic } \\
\text { Filling } \\
(n=40)\end{array}$ & $\begin{array}{c}\text { Grade } 1 \\
\text { Diastolic } \\
\text { Dysfunction } \\
(n=21)\end{array}$ & $\begin{array}{c}\text { Grade } 2 \\
\text { Diastolic } \\
\text { Dysfunction } \\
(n=45)\end{array}$ & $\begin{array}{c}\text { Grade } 3 \\
\text { Diastolic } \\
\text { Dysfunction } \\
(\mathrm{n}=9)\end{array}$ & $\mathbf{P}$ \\
\hline \multicolumn{6}{|l|}{$\overline{\text { CLINICAL }}$} \\
\hline Age (year) & $50 \pm 10$ & $62 \pm 7$ & $61 \pm 14$ & $52 \pm 12$ & $<0.001$ \\
\hline Male gender & $90 \%(36)$ & $86 \%(18)$ & $71 \%(32)$ & $100 \%(9)$ & $0.05^{*}$ \\
\hline \multicolumn{6}{|l|}{ Atherosclerosis Risk Factors } \\
\hline Hypertension & $28 \%(11)$ & $43 \%(9)$ & $62 \%(28)$ & $22 \%(2)$ & 0.007 \\
\hline Hyperlipidemia & $33 \%(13)$ & $38 \%(8)$ & $60 \%(27)$ & $56 \%(5)$ & $0.06^{*}$ \\
\hline Diabetes Mellitus & $18 \%(7)$ & $10 \%(2)$ & $27 \%(12)$ & $22 \%(2)$ & 0.41 \\
\hline Tobacco Use & $40 \%(16)$ & $24 \%(5)$ & $40 \%(18)$ & $33 \%(3)$ & 0.58 \\
\hline Family History & $38 \%(15)$ & $24 \%(5)$ & $18 \%(8)$ & $22 \%(3)$ & 0.22 \\
\hline \multicolumn{6}{|l|}{ Coronary Artery Disease History } \\
\hline Prior Myocardial Infarction & $3 \%(1)$ & $14 \%(3)$ & $4 \%(2)$ & $0 \%(-)$ & 0.20 \\
\hline Prior Coronary Revascularization & $5 \%(2)$ & $19 \%(4)$ & $11 \%(5)$ & $11 \%(1)$ & 0.40 \\
\hline \multicolumn{6}{|l|}{ Cardiovascular Medications } \\
\hline Beta-blocker & $100 \%(40)$ & $100 \%(21)$ & $98 \%(44)$ & $100 \%(9)$ & 0.67 \\
\hline ACE Inhibitor/ARB & $73 \%(29)$ & $62 \%(13)$ & $82 \%(37)$ & $67 \%(6)$ & 0.33 \\
\hline HMG-CoA Reductase Inhibitor & $98 \%(39)$ & $100 \%(21)$ & $93 \%(42)$ & $100 \%(9)$ & .47 \\
\hline Aspirin & $100 \%(40)$ & $100 \%(21)$ & $100 \%(45)$ & $100 \%(9)$ & - \\
\hline Thienopyridines & $98 \%(39)$ & $95 \%(20)$ & $93 \%(42)$ & $100 \%(9)$ & 0.72 \\
\hline \multicolumn{6}{|l|}{ Infarct Related Artery } \\
\hline Left Anterior Descending & $58 \%(23)$ & $62 \%(13)$ & $64 \%(29)$ & $78 \%(7)$ & 0.71 \\
\hline Right Coronary & $33 \%(13)$ & $24 \%(5)$ & $29 \%(13)$ & $11 \%(1)$ & 0.60 \\
\hline Left Circumflex & $10 \%(4)$ & $14 \%(3)$ & $7 \%(3)$ & $0 \%(-)$ & 0.57 \\
\hline \multicolumn{6}{|l|}{ Revascularization Strategy } \\
\hline Primary Percutaneous Intervention & $73 \%(29)$ & $62 \%(13)$ & $67 \%(30)$ & $56 \%(5)$ & 0.72 \\
\hline Primary Thrombolysis & $25 \%(10)$ & $43 \%(9)$ & $27 \%(12)$ & $44 \%(4)$ & 0.36 \\
\hline \multicolumn{6}{|l|}{ ECHOCARDIOGRAPHY } \\
\hline \multicolumn{6}{|l|}{ LV Morphology/Systolic Function } \\
\hline Ejection Fraction (\%) & $50 \pm 8$ & $50 \pm 11$ & $48 \pm 12$ & $32 \pm 15$ & $<0.001$ \\
\hline Wall Motion Score & $13 \pm 10$ & $14 \pm 11$ & $15 \pm 12$ & $33 \pm 14$ & $<0.001$ \\
\hline End-diastolic diameter (cm) & $5.6 \pm 0.5$ & $5.8 \pm 0.5$ & $5.6 \pm 0.5$ & $6.2 \pm 0.4$ & 0.01 \\
\hline End-systolic diameter (cm) & $4.1 \pm 0.5$ & $4.3 \pm 0.6$ & $4.2 \pm 0.6$ & $5.1 \pm 0.7$ & $<0.001$ \\
\hline Relative Wall Thickness $\left(\mathrm{g} / \mathrm{m}^{2}\right)$ & $.030 \pm 0.04$ & $0.29 \pm 0.03$ & $0.31 \pm 0.04$ & $0.25 \pm 0.04$ & 0.005 \\
\hline Myocardial Mass $\left(\mathrm{g} / \mathrm{m}^{2}\right)$ & $92 \pm 15$ & $97 \pm 14$ & $96 \pm 15$ & $92 \pm 16$ & 0.56 \\
\hline Left Atrial Diameter (cm) & $3.8 \pm 0.5$ & $3.9 \pm 0.6$ & $4.0 \pm 0.5$ & $4.4 \pm 0.4$ & 0.005 \\
\hline \multicolumn{6}{|c|}{ CARDIOVASCULAR MAGNETIC RESONANCE } \\
\hline \multicolumn{6}{|l|}{ LV Morphology/Systolic Function } \\
\hline Ejection fraction (\%) & $57 \pm 8$ & $55 \pm 9$ & $54 \pm 12$ & $41 \pm 10$ & 0.001 \\
\hline Wall motion Score & $11 \pm 9$ & $14 \pm 7$ & $15 \pm 10$ & $29 \pm 12$ & $<0.001$ \\
\hline End-diastolic volume (ml) & $151 \pm 34$ & $141 \pm 32$ & $149 \pm 45$ & $220 \pm 49$ & $<0.001$ \\
\hline End-systolic volume (ml) & $66 \pm 24$ & $66 \pm 26$ & $70 \pm 36$ & $133 \pm 49$ & $<0.001$ \\
\hline Myocardial Mass $\left(\mathrm{g} / \mathrm{m}^{2}\right)$ & $67 \pm 12$ & $71 \pm 14$ & $72 \pm 17$ & $79 \pm 20$ & 0.15 \\
\hline LV Infarct Size (\% myocardium) & $14 \pm 9$ & $16 \pm 9$ & $17 \pm 10$ & $27 \pm 8$ & 0.005 \\
\hline Left Atrial Volume (ml) & $80 \pm 19$ & $77 \pm 20$ & $95 \pm 31$ & $115 \pm 25$ & $<0.001$ \\
\hline
\end{tabular}

Boldface type indicates $p$ value $<0.05$

* indicates $p<0.1$ 
Table 2 Diastolic Filling Parameters

\begin{tabular}{|c|c|c|c|c|c|}
\hline & $\begin{array}{c}\text { Normal Diastolic } \\
\text { Filling } \\
(n=40)\end{array}$ & $\begin{array}{c}\text { Grade } 1 \\
\text { Diastolic Dysfunction } \\
(\mathrm{n}=21)\end{array}$ & $\begin{array}{c}\text { Grade } 2 \\
\text { Diastolic Dysfunction } \\
(\mathrm{n}=45)\end{array}$ & $\begin{array}{c}\text { Grade } 3 \\
\text { Diastolic Dysfunction } \\
(\mathrm{n}=9)\end{array}$ & $\mathbf{P}$ \\
\hline \multicolumn{6}{|l|}{ Cardiovascular Magnetic Resonance } \\
\hline E:A Ratio & $3.1 \pm 2.4$ & $1.1 \pm 0.4$ & $1.9 \pm 1.4$ & $4.8 \pm 3.3$ & $<0.001$ \\
\hline Diastolic Volume Recovery (\% diastole) & $65 \pm 16$ & $81 \pm 5$ & $73 \pm 13$ & $58 \pm 15$ & $<0.001$ \\
\hline Time to Peak Filling Rate (msec) & $174 \pm 119$ & $321 \pm 205$ & $221 \pm 136$ & $136 \pm 36$ & 0.001 \\
\hline Peak Filling Rate (ml/sec) & $266 \pm 76$ & $207 \pm 58$ & $231 \pm 73$ & $353 \pm 91$ & $<0.001$ \\
\hline Normalized Peak Filling Rate & $3.3 \pm 1.2$ & $2.7 \pm 0.6$ & $3.0 \pm 0.7$ & $4.1 \pm 0.8$ & 0.001 \\
\hline \multicolumn{6}{|l|}{ Echocardiography } \\
\hline E:A Ratio & $1.4 \pm 0.4$ & $0.9 \pm 0.3$ & $1.3 \pm 0.3$ & $3.1 \pm 1.2$ & $<0.001$ \\
\hline Deceleration time (msec) & $187 \pm 33$ & $249 \pm 41$ & $185 \pm 40$ & $127 \pm 16$ & $<0.001$ \\
\hline Isovolumic relaxation time (msec) & $90 \pm 12$ & $96 \pm 15$ & $89 \pm 17$ & $73 \pm 11$ & 0.02 \\
\hline Tissue Doppler e'/a' (septal) & $1.1 \pm 0.3$ & $0.6 \pm 0.2$ & $0.8 \pm 0.3$ & $1.2 \pm 0.5$ & $<0.001$ \\
\hline Tissue Doppler e'/a' (lateral) & $1.8 \pm 0.6$ & $0.8 \pm 0.3$ & $1.0 \pm 0.5$ & $1.9 \pm 0.6$ & $<0.001$ \\
\hline E/e' (mean e') & $6.3 \pm 2.0$ & $7.8 \pm 2.6$ & $9.5 \pm 2.6$ & $15.0 \pm 8.9$ & $<0.001$ \\
\hline
\end{tabular}

generated by LV-METRIC in relation to graded severity of echo-evidenced DD.

Repeat LV-METRIC processing was performed in 30 sequential patients to assess reproducibility (Table 3 ). All diastolic variables were highly reproducible, with $\leq 1 \%$ difference between readers for $D R_{80}$, TPFR, and PFR. Mean processing time was 2:09 \pm 0:51 minutes for the initial reader $(175 \pm 34$ images per exam), with similar processing time $(2: 15 \pm 0: 50)$ for the second reader $(\mathrm{p}=0.12)$.

Impact of Diastolic Dysfunction on Left Ventricular Filling Table 2 reports CMR and echo diastolic parameters for patients stratified according to graded severity of DD, demonstrating similar patterns for the two modalities. Figure 3 provides a side-by-side comparison of echo and CMR-evidenced diastolic filling parameters (Figure 3A) and timing intervals (Figure $3 \mathrm{~B}$ ) stratified by graded DD severity. Whereas E/e' by echo and PFR by cine-CMR (Figure 3A) increased with transition from grade 1 through grade $3 \mathrm{DD}$, deceleration time and $\mathrm{DVR}_{80}$ (Figure 3B) manifested a biphasic relationship - increasing among patients with grade 1 and decreasing among patients with grade 2 and 3 DD. Among the overall population, $\mathrm{DVR}_{80}$ modestly correlated with echoevidenced deceleration time $(\mathrm{r}=0.25, \mathrm{p}=0.01)$.

\section{E:A Profiles}

E:A filling ratios by CMR and echo (Table 2) also yielded similar patterns in relation to graded DD severity. For both modalities, ratios were diminished with grade 1 , progressively increased with grade 2 , and augmented with grade 3 DD. When classified by the standard of echo-assigned diastolic grade, E:A ratios by cine-CMR were lower among patients with grade $1 \mathrm{DD}$ compared to those with normal diastolic filling (1.1 \pm 0.4 vs. $3.1 \pm 2.4, \mathrm{p}<0.001)$, reflecting a similar relationship for echo-derived E:A ratios between these groups $(0.9 \pm 0.3$ vs. $1.4 \pm 0.4, p<0.001)$. Conversely, when patients with grade 3 DD were compared to normals, E: A ratios by cine-CMR tended to be higher among the DD group ( $4.8 \pm 3.3$ vs. $3.1 \pm 2.4, \mathrm{p}=0.099)$, again paralleling echo-derived E:A ratios $(3.1 \pm 1.2$ vs. $1.4 \pm 0.4$, $\mathrm{p}<0.01)$.

Among patients with echo-evidenced grade 2 DD, cine-CMR demonstrated E:A ratios > 1 in 68\% (28/41; $n$ $=4$ with non-discernable E:A profiles). For echo, magnitude of E:A ratios was similar between patients with grade $2 \mathrm{DD}$ and normal diastolic filling $(1.3 \pm 0.3$ vs. 1.4 $\pm 0.4, \mathrm{p}=0.16)$. For cine-CMR, E:A ratios were slightly lower among the DD group $(1.9 \pm 1.4$ vs. $3.1 \pm 2.4, \mathrm{p}<$ $0.01)$, consistent with decreased volumetric filling during

Table 3 Reproducibility of CMR Indices*

\begin{tabular}{|c|c|c|c|c|c|c|c|}
\hline & \multirow[b]{2}{*}{ Initial } & \multicolumn{3}{|c|}{ Intra-Observer Reproducibility } & \multicolumn{3}{|c|}{ Inter-Observer Reproducibility } \\
\hline & & Repeat & $\Delta \pm \mathrm{SD}$ & $p$ & Repeat & $\Delta \pm \mathrm{SD}$ & $p$ \\
\hline Diastolic Volume Recovery (\%) & $69 \pm 15$ & $71 \pm 15$ & $1.5 \pm 5.6$ & 0.16 & $69 \pm 16$ & $0.2 \pm 1.7$ & 0.58 \\
\hline Time to Peak Filling Rate (msec) & $231 \pm 177$ & $232 \pm 176$ & $1.3 \pm 6.9$ & 0.33 & $233 \pm 179$ & $1.5 \pm 8.4$ & 0.33 \\
\hline Peak Filling Rate (ml/sec) & $243 \pm 91$ & $245 \pm 94$ & $2.2 \pm 10.1$ & 0.25 & $246 \pm 92$ & $2.8 \pm 11.5$ & 0.19 \\
\hline E/A Ratio & $2.5 \pm 1.9$ & $2.5 \pm 1.9$ & $0.1 \pm 0.7$ & 0.52 & $2.9 \pm 2.3$ & $0.4 \pm 1.5$ & 0.17 \\
\hline Processing Time (minutes) & $2: 09 \pm 0: 51$ & $2: 06 \pm 0: 46$ & $0: 03 \pm 0: 17$ & 0.28 & $2: 15 \pm 0: 50$ & $0: 06 \pm 0: 21$ & 0.12 \\
\hline
\end{tabular}

* reproducibility and processing times assessed in subgroup of 30 consecutive patients 
A
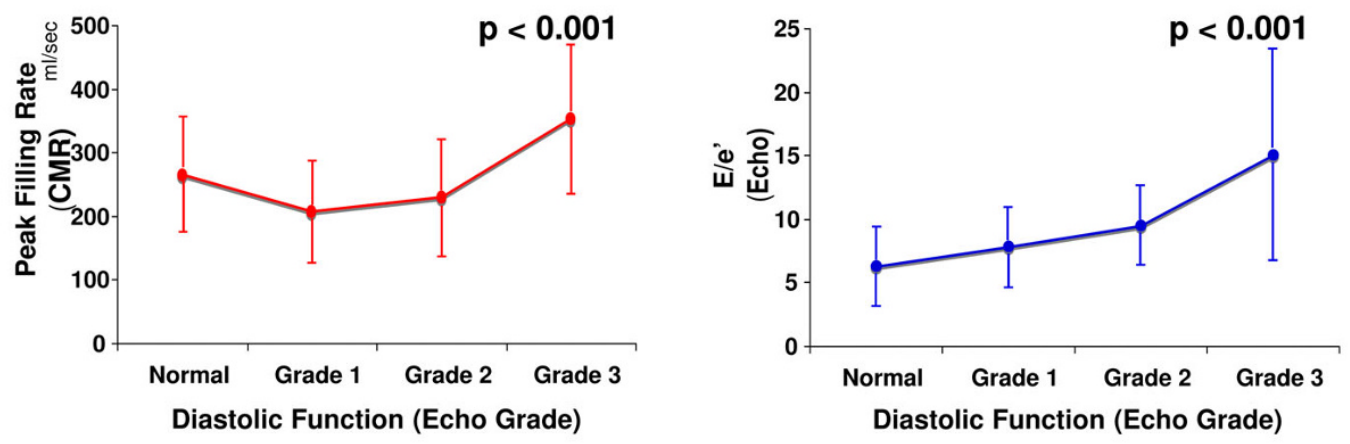

B
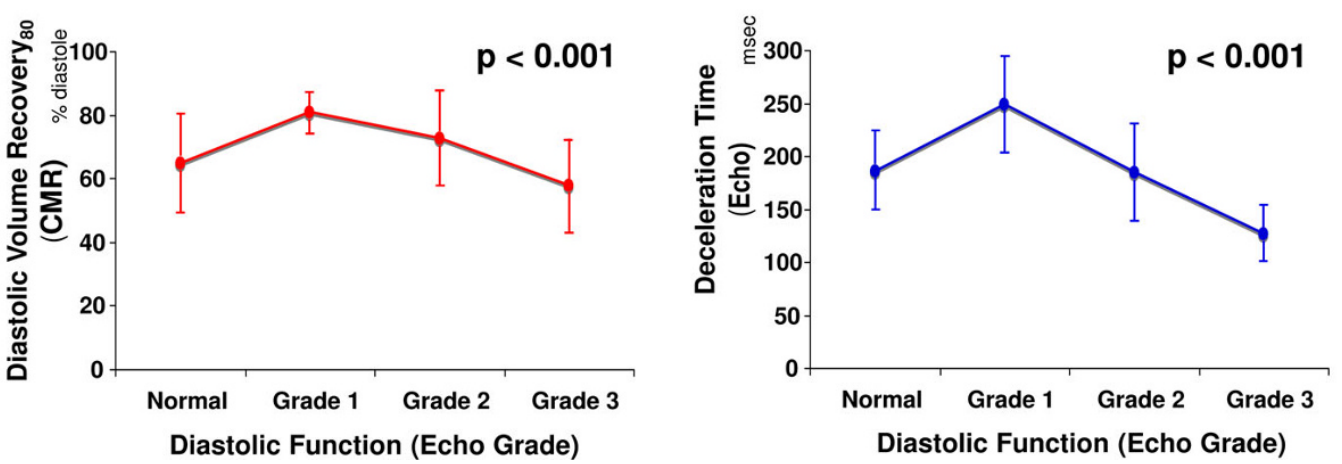

Figure 3 Diastolic Filling Indices in Relation to Severity of Diastolic Dysfunction. Diastolic indices by cine-CMR (red) and echo (blue) stratified by echo-assigned diastolic grade. (A) PFR by cine-CMR paralleled E/e' by echo. (B) DVR 80 by cine-CMR paralleled echo-evidenced deceleration time (data presented as mean \pm SD).

early diastole. As further evidence of impaired early diastolic filling, patients with grade $2 \mathrm{DD}$ had longer $\mathrm{DVR}_{80}(73 \pm 13$ vs. $65 \pm 16, \mathrm{p}=0.01)$ and lower PFR $(231 \pm 73$ vs. $266 \pm 76, \mathrm{p}=0.04)$ when compared to normals.

\section{Diastolic Performance of cine-CMR Indices}

To evaluate diagnostic performance of CMR indices in relation to echo DD severity, parameters were tested among each discrete group of echo-assigned diastolic grade and the common group of patients with normal echo-evidenced diastolic filling. For $\mathrm{DVR}_{80}$, the previously established normative threshold of $<77 \%$ diastole (to recover $80 \%$ stroke volume) was applied based on our prior research among controls with normal systolic/diastolic function [15]. For all other CMR indices, diagnostic thresholds were determined using a cutoff necessary to achieve a matched specificity $(83 \%)$ to $\mathrm{DVR}_{80}$.

Diagnostic performance of CMR indices varied according to graded severity of echo-evidenced DD.

Table 4 Performance of cine-CMR Indices in Relation to Diastolic Dysfunction Severity - Diastolic Filling Intervals

\begin{tabular}{|c|c|c|c|c|c|c|}
\hline & Threshold & Sensitivity & Specificity & Accuracy & $\begin{array}{c}\text { Positive } \\
\text { Predictive } \\
\text { Value } \\
\end{array}$ & $\begin{array}{c}\text { Negative } \\
\text { Predictive } \\
\text { Value }\end{array}$ \\
\hline \multicolumn{7}{|l|}{ GRADE 1 (mild) } \\
\hline Diastolic Volume Recovery 80 & $77 \% *$ & $71 \%(15 / 21)$ & $83 \%(33 / 40)$ & $79 \%(48 / 61)$ & $68 \%(15 / 22)$ & $85 \%(33 / 39)$ \\
\hline Time to Peak Filling Rate & $221 \mathrm{msect}$ & $48 \%(10 / 21)$ & $83 \%(33 / 40)$ & $70 \%(43 / 61)$ & $59 \%(10 / 17)$ & $75 \%(33 / 44)$ \\
\hline \multicolumn{7}{|l|}{ GRADE 2 (moderate) } \\
\hline Diastolic Volume Recovery 80 & $77 \%$ & $47 \%(21 / 45)$ & $83 \%(33 / 40)$ & $64 \%(54 / 85)$ & $75 \%(21 / 28)$ & $58 \%(33 / 57)$ \\
\hline Time to Peak Filling Rate & $221 \mathrm{msec}$ & $29 \%(13 / 45)$ & $83 \%(33 / 40)$ & $54 \%(46 / 85)$ & $65 \%(13 / 20)$ & $51 \%(33 / 65)$ \\
\hline \multicolumn{7}{|l|}{ GRADE 3 (severe) } \\
\hline Diastolic Volume Recovery 80 & $77 \%$ & $0 \%(0 / 9)$ & $83 \%(33 / 40)$ & $67 \%(33 / 49)$ & $0 \%(0 / 7)$ & $79 \%(33 / 42)$ \\
\hline Time to Peak Filling Rate & $221 \mathrm{msec}$ & $0 \%(0 / 9)$ & $83 \%(33 / 40)$ & $67 \%(33 / 49)$ & $0 \%(0 / 7)$ & $79 \%(33 / 42)$ \\
\hline
\end{tabular}


Table 5 Performance of cine-CMR Indices in Relation to Diastolic Dysfunction Severity - Diastolic Filling Rates

\begin{tabular}{|c|c|c|c|c|c|c|}
\hline & Threshold & Sensitivity & Specificity & Accuracy & $\begin{array}{c}\text { Positive } \\
\text { Predictive } \\
\text { Value }\end{array}$ & $\begin{array}{c}\text { Negative } \\
\text { Predictive } \\
\text { Value }\end{array}$ \\
\hline \multicolumn{7}{|l|}{ GRADE 1 (mild) } \\
\hline Peak Filling Rate & $344 \mathrm{ml} / \mathrm{sect}$ & $5 \%(1 / 21)$ & $83 \%(33 / 40)$ & $56 \%(34 / 61)$ & $13 \%(1 / 8)$ & $62 \%(33 / 53)$ \\
\hline Normalized Peak Filling Rate & $4.02 / \mathrm{sect}$ & $0 \%(0 / 21)$ & $83 \%(33 / 40)$ & $54 \%(33 / 61)$ & $0 \%(0 / 7)$ & $61 \%(33 / 54)$ \\
\hline \multicolumn{7}{|l|}{ GRADE 2 (moderate) } \\
\hline Peak Filling Rate & $344 \mathrm{ml} / \mathrm{sec}$ & $7 \%(3 / 45)$ & $83 \%(33 / 40)$ & $42 \%(36 / 85)$ & $30 \%(3 / 10)$ & $44 \%(33 / 75)$ \\
\hline Normalized Peak Filling Rate & $4.02 / \mathrm{sec}$ & $9 \%(4 / 45)$ & $83 \%(33 / 40)$ & $44 \%(37 / 85)$ & $36 \%(4 / 11)$ & $45 \%(33 / 74)$ \\
\hline \multicolumn{7}{|l|}{ GRADE 3 (severe) } \\
\hline Peak Filling Rate & $344 \mathrm{ml} / \mathrm{sec}$ & $56 \%(5 / 9)$ & $83 \%(33 / 40)$ & $78 \%(38 / 49)$ & $42 \%(5 / 12)$ & $89 \%(33 / 37)$ \\
\hline Normalized Peak Filling Rate & $4.02 / \mathrm{sec}$ & $67 \%(6 / 9)$ & $83 \%(33 / 40)$ & $80 \%(39 / 49)$ & $46 \%(6 / 13)$ & $91 \%(33 / 36)$ \\
\hline
\end{tabular}

* Threshold of $77 \%$ diastole (to recover $80 \%$ stroke volume) based on previously established normative cutoff [15]

† Matched to achieve equivalent specificity vs. DVR 80

DVR $_{80}$ yielded a sensitivity of $71 \%$ for patients with grade 1 but did not identify any patients with grade 3 DD (Table 4). Conversely, at a matched specificity of $83 \%$, stroke volume adjusted nPFR yielded a sensitivity of $67 \%$ for grade 3 but did not identify any patients with grade 1 DD, with similar results for unadjusted PFR (Table 5). Whereas PFR or $\mathrm{DVR}_{80}$ alone identified fewer than half of patients with echo-evidenced grade $2 \mathrm{DD}$, use of the two parameters as aggregate criteria identified $53 \%(24 / 45)$ of affected patients.

Consistent with diagnostic performance results, multinomial logistic regression demonstrated that prolonged diastolic filling intervals and increased diastolic filling rates were associated with different aspects of echoevidenced diastolic dysfunction. As shown in Table 6, prolonged $\mathrm{DVR}_{80}$ was associated with presence of grade 1 (OR 2.79 per 10 point increment, CI 1.65-4.05, $\mathrm{p}=0.001$ ) with a similar trend for grade 2 (OR 1.35, CI $0.98-1.74, \mathrm{p}=0.06$ ), whereas high PFR was associated with grade 3 DD (OR 1.14 per $10 \mathrm{ml} / \mathrm{sec}$, CI 1.02-1.25, $\mathrm{p}=0.02)$.

\section{Discussion}

This study demonstrates that automated processing of routine cine-CMR can discern physiologic changes that occur with graded severity of DD. LV-METRIC generated LV filling curves within an average processing time of 2 minutes and yielded highly reproducible indices. Diastolic filling ratios (E:A), timing intervals $\left(\mathrm{DVR}_{80}\right)$, and filling rates (PFR) paralleled echo findings relative to $\mathrm{DD}$ grade. Diastolic filling $\left(\mathrm{DVR}_{80}\right)$ was prolonged in patents with grade 1 and shortened with grade $3 \mathrm{DD}$, paralleling echo-evidenced DT. Diastolic filling rates (PFR, nPFR) increased with DD grade, similar to E/e' by echo. At matched specificities of $83 \%, \mathrm{DVR}_{80}$ identified $71 \%$ of patients with grade 1 but no patients with grade 3 DD, while nPFR identified a similar proportion (67\%) with grade 3 but no patient with grade $1 \mathrm{DD}$.

Diastolic indices generated by LV-METRIC were concordant with established concepts regarding filling changes that occur with graded DD severity $[4,22,23]$. For grade 1, impaired LV relaxation results in decreased early (E) filling, increased atrial (A) filling, and

Table 6 Functional CMR Predictors of Diastolic Dysfunction Severity

\begin{tabular}{|c|c|c|c|c|}
\hline & Variable & $\begin{array}{l}\text { Odds } \\
\text { Ratio }\end{array}$ & $\begin{array}{l}\text { 95\% Confidence } \\
\text { Interval }\end{array}$ & $P$ \\
\hline \multicolumn{5}{|l|}{ GRADE 1 (mild) } \\
\hline & Diastolic Volume Recovery $80^{*}$ & 2.79 & $1.65-4.05$ & 0.001 \\
\hline & Peak Filling Rate† & 0.95 & $0.86-1.04$ & 0.28 \\
\hline \multicolumn{5}{|c|}{ GRADE 2 (moderate) } \\
\hline & Diastolic Volume Recovery80 & 1.35 & $0.98-1.74$ & 0.06 \\
\hline & Peak Filling Rate & 0.96 & $0.89-1.03$ & 0.24 \\
\hline \multicolumn{5}{|l|}{ GRADE 3 (severe) } \\
\hline & Diastolic Volume Recovery80 & 1.00 & $0.51-1.51$ & 1.00 \\
\hline & Peak Filling Rate & 1.14 & $1.02-1.25$ & 0.02 \\
\hline
\end{tabular}

Model $\chi^{2}=43.3, \mathrm{p}<0.001$

* Per 10 point increment

† Per $10 \mathrm{ml} / \mathrm{sec}$ increment 
prolonged time from end-systole to peak diastolic filling. For grade 2 (pseudonormalization), the combination of impaired LV relaxation, decreased compliance, and increased LA pressure results in a seemingly normal E:A filling pattern. For grade 3 (restrictive filling), impaired LV relaxation and compliance are offset by a marked increase in LA pressure, producing an increased E:A ratio with an increased filling rate but shortened duration of early LV filling and a reduction in late diastolic filling. Our results were consistent with these physiologic concepts. Data generated by LV-METRIC demonstrated a decrease in CMR E:A ratio $(1.1 \pm 0.4)$ for patients with grade 1 , and stepwise increase among patients with grade $2(1.9 \pm 1.4)$ and grade $3(4.8 \pm 3.3)$ $(\mathrm{p}<0.001)$. Diastolic filling intervals $\left(\mathrm{DVR}_{80}, \mathrm{TPFR}\right)$ were prolonged in grade 1 and correlated with echoevidenced DT. PFR was increased and TPFR decreased among patients with grade $3 \mathrm{DD}$, consistent with augmented early diastolic filling in restrictive physiology.

LV filling indices derived from invasive angiography and radionuclide imaging have been used to assess diastolic performance [7-10,24]. Consistent with our cineCMR results, invasive studies have shown that PFR is decreased in impaired LV relaxation and increased with progressive LV stiffness and augmented LA-LV diastolic filling gradients. This concept was demonstrated by Ohno et al, who used a pacing-induced heart failure model to demonstrate that peak early diastolic filling rate initially decreases and subsequently increases with progressive heart failure [25]. In this study, peak filling rate correlated with increased LA-LV filling gradients and was inversely related to LV stiffness. PFR measured invasively has also been shown to correlate with diastolic atrio-ventricular filling gradients and LV relaxation rates [26]. Studies using radionuclide angiography have reported reduced PFR, increased TPFR, and prolonged early filling intervals among coronary artery disease patients with preserved systolic function, suggesting that these changes may reflect one aspect of DD $[27,28]$.

Our results extend upon prior studies that have demonstrated feasibility of DD assessment by cineCMR. Maceira et al reported age-associated decrements in PFR among a normative cohort [29]. However, as this study was restricted to asymptomatic subjects without cardiac disease, normal and abnormal diastolic indices were not compared. Other studies have reported differences in PFR and TPFR among patients grouped by binary presence or absence of echo-evidenced DD [15,30]. However, these studies did not examine relations between DD severity and cine-CMR indices. Our study, conducted in a broad post-MI cohort with variable severity of DD, demonstrates that different aspects of LV filling by cine-CMR can be used to distinguish between grades of echo-evidenced DD. We are unaware of any prior study that has assessed the relation between graded severity of DD and global LV filling by any noninvasive imaging modality.

While multiple techniques can be used to assess LV filling, there are several potential advantages to cineCMR. First, unlike radionuclide imaging, CMR entails no ionizing radiation and is thereby well-suited for serial assessment of diastolic performance. Our results demonstrated that cine-CMR indices were highly reproducible, with $\leq 1 \%$ inter-reader differences for multiple parameters $\left(D R_{80}, T P F R, P F R\right)$. Second, while LV-METRIC is a novel segmentation algorithm, the actual cine-CMR data required is a standard component of nearly all clinical CMR exams and requires no tailored pulse sequences or imaging hardware. This enables our approach to be widely applied to both prospective and pre-existing exams. Our prior study demonstrated feasibility among a retrospective dataset of patients with normal systolic function [15]. Our current study extends upon this by demonstrating that LV-METRIC can discern diastolic filling changes among a broad population with varying severity of systolic dysfunction and diastolic impairment. Third, unlike echo, CMR can directly assess myocardial tissue composition and thereby assess the influence of infarcted myocardium on diastolic performance. Our results demonstrated a stepwise increase in infarct size with graded severity of DD, suggesting that changes in myocyte tissue composition may alter LV compliance and contribute to DD.

Several limitations of our study should be recognized. In our study, LV volumes were assessed using short axis images acquired with $6 \mathrm{~mm}$ slice thickness and $4 \mathrm{~mm}$ gap. It is possible that gaps between LV slices could impact volumetric assessment. Future studies incorporating high-resolution 3D cine-CMR for volumetric assessment of LV diastolic function are planned to address this issue. Additionally, while our findings demonstrate that automated processing of routine cineCMR can discern filling changes that occur with increasing severity of DD, approximately $1 / 3$ of patients with grade 1 or grade 3 , and nearly $1 / 2$ of patents with grade 2 DD were not classified as abnormal by cineCMR. This may be attributable to differences in temporal resolution between modalities and it is possible that diagnostic results would have been better if dedicated, higher temporal resolution cine-CMR were performed. However, prior studies have reported that LV filling indices generated by high resolution RNCA were discordant with echo in $16 \%$ of cases, suggesting that differences between modalities in our study are partially attributable to the approach used for diastolic assessment [31]. Our segmentation approach focused solely on LV filling, which may be limited if the LA compensates to preserve normal LV diastolic filling. This may 
be addressed through LA segmentation, which would be particularly useful to identify physiologic changes in grade $2 \mathrm{DD}$. As LV-METRIC utilizes routine cine-CMR data, future applications may combine LV and LA segmentation for integrated assessment of diastolic performance.

\section{Conclusions}

In conclusion, this study demonstrates that LV filling curves generated by automated processing of routine cine-CMR can be used to discern volumetric filling changes that occur with graded severity of DD. Patients with grade 1-2 had prolonged filling intervals whereas patients with grade 3 dysfunction had increased diastolic filling rates. Future studies are needed to ascertain whether cine-CMR indices can be used to guide therapeutic management and stratify clinical outcomes for patients at risk for DD and related complications.

\section{Abbreviations}

DD: diastolic dysfunction; CMR: cardiovascular magnetic resonance; PFR: peak filling rate (absolute); nPFR: normalized peak filling rate (stroke volume adjusted); TPFR: time to peak filling rate; DVR: diastolic volume recovery;

\section{Acknowledgements}

Funding for this study was provided by the Doris Duke Clinical Scientist Development Award (JWW), AOA Research Fellowship (SJM), Lantheus Medical Imaging (clinical trial \#NCT00539045), and Weill Cornell Clinical Translational Science Center.

\section{Author details}

'Division of Cardiology, Department of Medicine, Weill Cornell Medical College, NY, NY, USA. ${ }^{2}$ Department of Radiology, Weill Cornell Medical College, NY, NY, USA.

\section{Authors' contributions \\ DDM participated in the study's design and coordination, image analysis, data collection, and manuscript preparation. NCFC and YW developed the automated CMR segmentation software (LV-METRIC) used for image analysis. SS, KK, SJM, JKM, TML, MRP and RBD contributed to image/data analysis and study design. JWW conceived of the study, participated in its design and coordination, and drafted the manuscript. All authors read and approved the final manuscript. \\ Competing interests \\ The authors' institution has submitted a patent for the automated segmentation algorithm (LV-METRIC) described in this study.}

Received: 31 March 2010 Accepted: 31 July 2010

Published: 31 July 2010

\section{References}

1. Moller JE, Sondergaard E, Poulsen SH, Egstrup K: Pseudonormal and restrictive filling patterns predict left ventricular dilation and cardiac death after a first myocardial infarction: a serial color M-mode Doppler echocardiographic study. J Am Coll Cardiol 2000, 36:1841-6.

2. Poulsen $\mathrm{SH}$, Jensen SE, Egstrup K: Longitudinal changes and prognostic implications of left ventricular diastolic function in first acute myocardial infarction. Am Heart J 1999, 137:910-8.

3. Moller JE, Whalley GA, Dini FL, Doughty RN, Gamble GD, Klein AL, Quintana M, Yu CM: Independent prognostic importance of a restrictive left ventricular filling pattern after myocardial infarction: an individual patient meta-analysis: Meta-Analysis Research Group in
Echocardiography acute myocardial infarction. Circulation 2008, 117:2591-8.

4. Nagueh SF, Appleton CP, Gillebert TC, Marino PN, Oh JK, Smiseth OA Waggoner AD, Flachskampf FA, Pellikka PA, Evangelista A:

Recommendations for the evaluation of left ventricular diastolic function by echocardiography. J Am Soc Echocardiogr 2009, 22:107-33.

5. Feigenbaum H: Feigenbaum's Echocardiography Philadelphia: Lippincott Williams \& Wilkins, 61996.

6. Ommen SR, Nishimura RA, Appleton CP, Miller FA, Oh JK, Redfield MM Tajik AJ: Clinical utility of Doppler echocardiography and tissue Doppler imaging in the estimation of left ventricular filling pressures: $A$ comparative simultaneous Doppler-catheterization study. Circulation 2000, 102:1788-94.

7. Magorien DJ, Shaffer P, Bush CA, Magorien RD, Kolibash AJ, Leier CV, Bashore TM: Assessment of left ventricular pressure-volume relations using gated radionuclide angiography, echocardiography, and micromanometer pressure recordings. A new method for serial measurements of systolic and diastolic function in man. Circulation 1983, 67:844-53

8. Villari B, Betocchi S, Pace L, Piscione F, Russolillo E, Ciarmiello A, Salvatore M, Condorelli M, Chiariello M: Assessment of left ventricular diastolic function: comparison of contrast ventriculography and equilibrium radionuclide angiography. J Nucl Med 1991, 32:1849-53.

9. Muntinga HJ, van den Berg F, Knol HR, Niemeyer MG, Blanksma PK, Louwes $\mathrm{H}$, van der Wall EE: Normal values and reproducibility of left ventricular filling parameters by radionuclide angiography. Int J Card Imaging 1997, 13:165-71, discussion 173.

10. Akincioglu C, Berman DS, Nishina H, Kavanagh PB, Slomka PJ, Abidov A, Hayes S, Friedman JD, Germano G: Assessment of diastolic function using 16-frame 99mTc-sestamibi gated myocardial perfusion SPECT: normal values. J Nucl Med 2005, 46:1102-8.

11. Dewey M, Muller M, Eddicks S, Schnapauff D, Teige F, Rutsch W, Borges AC, Hamm B: Evaluation of global and regional left ventricular function with 16-slice computed tomography, biplane cineventriculography, and twodimensional transthoracic echocardiography: comparison with magnetic resonance imaging. J Am Coll Cardiol 2006, 48:2034-44.

12. Heckbert SR, Post W, Pearson GD, Arnett DK, Gomes AS, Jerosch-Herold M, Hundley WG, Lima JA, Bluemke DA: Traditional cardiovascular risk factors in relation to left ventricular mass, volume, and systolic function by cardiac magnetic resonance imaging: the Multiethnic Study of Atherosclerosis. J Am Coll Cardiol 2006, 48:2285-92.

13. Codella NC, Weinsaft JW, Cham MD, Janik M, Prince MR, Wang Y: Left ventricle: automated segmentation by using myocardial effusion threshold reduction and intravoxel computation at MR imaging. Radiology 2008, 248:1004-12.

14. Codella NCF, Cham MD, Wong R, Chu C, Min JK, Prince MR, Wang Y, Weinsaft JW: Rapid and accurate left ventricular chamber quantification using a novel CMR segmentation algorithm: a clinical validation study. Journal of Magnetic Resonance Imaging 2010, 31:845-53.

15. Kawaji K, Codella NC, Prince MR, Chu CW, Shakoor A, LaBounty TM, Min JK, Swaminathan RV, Devereux RB, Wang Y, Weinsaft JW: Automated segmentation of routine clinical cardiac magnetic resonance imaging for assessment of left ventricular diastolic dysfunction. Circ Cardiovasc Imaging 2009, 2:476-84.

16. Azevedo CF, Amado LC, Kraitchman DL, Gerber BL, Osman NF, Rochitte CE, Edvardsen T, Lima JA: Persistent diastolic dysfunction despite complete systolic functional recovery after reperfused acute myocardial infarction demonstrated by tagged magnetic resonance imaging. Eur Heart J 2004, 25:1419-27.

17. Lang RM, Biereg M, Devereux RM, Flachskampf FA, Foster E, Pellikka PA, Picard MA, Roman MJ, Seward S, Shanewise JS, Solomon SD, Spencer KT, St John Sutton M, Stewart WJ: Recommendations for Chamber Quantification: A Report from the American Society of Echocardiography's Guidelines and Standards Committee and the Chamber Quantification Writing Group, Developed in Conjunction with the European Association of Echocardiography, a Branch of the European Society of Cardiology. J Am Soc Echocardiogr 2005, 18:1440-1463. 
18. Akkan D, Kjaergaard J, Moller JE, Hassager C, Torp-Pedersen C, Kober L: Prognostic importance of a short deceleration time in symptomatic congestive heart failure. Eur J Heart Fail 2008, 10:689-95.

19. Poulsen $\mathrm{SH}$, Andersen $\mathrm{NH}$, Ivarsen $\mathrm{Pl}$, Mogensen $\mathrm{CE}$, Egeblad H: Doppler tissue imaging reveals systolic dysfunction in patients with hypertension and apparent "isolated" diastolic dysfunction. J Am Soc Echocardiogr 2003, 16:724-31

20. Lee HY, Codella N, Cham M, Weinsaft J, Wang Y: Automatic Left Ventricle Segmentation Using Iterative Thresholding and Active Contour Model With Adaptation on Short-Axis Cardiac MRI. IEEE Trans Biomed Eng 2009.

21. Sievers B, Elliott MD, Hurwitz LM, Albert TS, Klem I, Rehwald WG, Parker MA, Judd RM, Kim RJ: Rapid detection of myocardial infarction by subsecond, free-breathing delayed contrast-enhancement cardiovascular magnetic resonance. Circulation 2007, 115:236-44

22. Zile MR, Brutsaert DL: New concepts in diastolic dysfunction and diastolic heart failure: Part I: diagnosis, prognosis, and measurements of diastolic function. Circulation 2002, 105:1387-93.

23. Redfield MM, Jacobsen SJ, Burnett JC Jr, Mahoney DW, Bailey KR, Rodeheffer RJ: Burden of systolic and diastolic ventricular dysfunction in the community: appreciating the scope of the heart failure epidemic. JAMA 2003, 289:194-202.

24. Hammermeister KE, Warbasse JR: The rate of change of left ventricular volume in man. II. Diastolic events in health and disease. Circulation 1974, 49:739-47.

25. Ohno M, Cheng CP, Little WC: Mechanism of altered patterns of left ventricular filling during the development of congestive heart failure. Circulation 1994, 89:2241-50.

26. Ishida Y, Meisner JS, Tsujioka K, Gallo Jl, Yoran C, Frater RW, Yellin EL: Left ventricular filling dynamics: influence of left ventricular relaxation and left atrial pressure. Circulation 1986, 74:187-96.

27. Bonow RO, Bacharach SL, Green MV, Kent KM, Rosing DR, Lipson LC, Leon MB, Epstein SE: Impaired left ventricular diastolic filling in patients with coronary artery disease: assessment with radionuclide angiography. Circulation 1981, 64:315-23.

28. Reduto LA, Wickemeyer WJ, Young JB, Del Ventura LA, Reid JW, Glaeser DH, Quinones MA, Miller RR: Left ventricular diastolic performance at rest and during exercise in patients with coronary artery disease. Assessment with first-pass radionuclide angiography. Circulation 1981, 63:1228-37.

29. Maceira AM, Prasad SK, Khan M, Pennell DJ: Normalized left ventricular systolic and diastolic function by steady state free precession cardiovascular magnetic resonance. J Cardiovasc Magn Reson 2006, 8:417-26.

30. Kudelka AM, Turner DA, Liebson PR, Macioch JE, Wang JZ, Barron JT: Comparison of cine magnetic resonance imaging and Doppler echocardiography for evaluation of left ventricular diastolic function. Am J Cardiol 1997, 80:384-6.

31. Spirito P, Maron BJ, Bonow RO: Noninvasive assessment of left ventricular diastolic function: comparative analysis of Doppler echocardiographic and radionuclide angiographic techniques. J Am Coll Cardiol 1986, 7:518-26.

doi:10.1186/1532-429X-12-46

Cite this article as: Mendoza et al: Impact of diastolic dysfunction severity on global left ventricular volumetric filling - assessment by automated segmentation of routine cine cardiovascular magnetic resonance. Journal of Cardiovascular Magnetic Resonance 2010 12:46.

\section{Submit your next manuscript to BioMed Central and take full advantage of:}

- Convenient online submission

- Thorough peer review

- No space constraints or color figure charges

- Immediate publication on acceptance

- Inclusion in PubMed, CAS, Scopus and Google Scholar

- Research which is freely available for redistribution

Submit your manuscript at www.biomedcentral.com/submit 